\title{
In-feed bambermycin medication induces anti-inflammatory effects and prevents parietal cell loss without influencing Helicobacter suis colonization in the stomach of mice
}

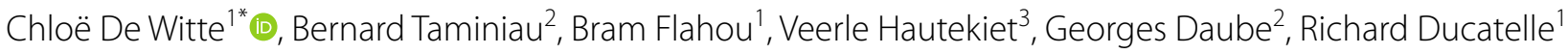 \\ and Freddy Haesebrouck ${ }^{1^{*}}$
}

\begin{abstract}
The minimum inhibitory concentration of bambermycin on three porcine Helicobacter suis strains was shown to be $8 \mu \mathrm{g} / \mathrm{mL}$. The effect of in-feed medication with this antibiotic on the course of a gastric infection with one of these strains, the host response and the gastric microbiota was determined in mice, as all of these parameters may be involved in gastric pathology. In H. suis infected mice which were not treated with bambermycin, an increased number of infiltrating B-cells, T-cells and macrophages in combination with a Th2 response was demonstrated, as well as a decreased parietal cell mass. Compared to this non-treated, infected group, in H. suis infected mice medicated with bambermycin, gastric $H$. suis colonization was not altered, but a decreased number of infiltrating T-cells, B-cells and macrophages as well as downregulated expressions of IL-1 $\beta, I L-8 \mathrm{M}, \mathrm{IL}-10$ and IFN- $\gamma$ were demonstrated and the parietal cell mass was not affected. In bambermycin treated mice that were not infected with $H$. suis, the number of infiltrating T-cells and expression of IL-1 $\beta$ were lower than in non-infected mice that did not receive bambermycin. Gastric microbiota analysis indicated that the relative abundance of bacteria that might exert unfavorable effects on the host was decreased during bambermycin supplementation. In conclusion, bambermycin did not affect $H$. suis colonization, but decreased gastric inflammation and inhibited the effects of a H. suis infection on parietal cell loss. Not only direct interaction of $H$. suis with parietal cells, but also inflammation may play a role in death of these gastric acid producing cells.
\end{abstract}

\section{Introduction}

Helicobacter suis is a Gram-negative, spiral-shaped bacterium found in the stomach of up to $90 \%$ of pigs at slaughter age, causing gastritis and a decreased daily weight gain $[1,2]$. In addition, infection with this bacterium has been associated with ulceration of the non-glandular part of the porcine stomach, most likely through induction of gastric acid secretion alterations and by influencing the gastric microbiota [3]. Apart from pigs, H. suis

\footnotetext{
*Correspondence: chloe.dewitte@ugent.be; freddy.haesebrouck@ugent.be ${ }^{1}$ Department of Pathology, Bacteriology and Avian Diseases, Faculty of Veterinary Medicine, Ghent University, Merelbeke, Belgium Full list of author information is available at the end of the article
}

is the most prevalent gastric non-H. pylori Helicobacter $(\mathrm{NHPH})$ species in humans. H. suis infection in humans has been associated with gastritis, peptic ulcers and mucosa-associated lymphoid tissue (MALT) lymphoma [4]. Direct or indirect contact with $H$. suis infected pigs or consumption of contaminated water or raw or undercooked pork may be a source of human infection [5]. Since rodent models are easily colonized with $H$. suis, the role of wild mice as vectors might also be considered.

In general, Helicobacter sp. infected human patients are treated with a combination of a proton pump inhibitor and two or three antibiotics selected from clarithromycin, amoxicillin, metronidazole, tetracycline and/ or levofloxacin [6]. A similar therapeutic protocol is not indicated in $H$. suis infected pigs since this is expensive,

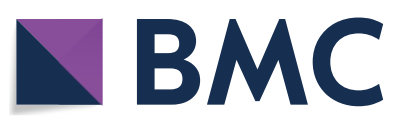

(c) The Author(s) 2018. This article is distributed under the terms of the Creative Commons Attribution 4.0 International License (http://creativecommons.org/licenses/by/4.0/), which permits unrestricted use, distribution, and reproduction in any medium, provided you give appropriate credit to the original author(s) and the source, provide a link to the Creative Commons license, and indicate if changes were made. The Creative Commons Public Domain Dedication waiver (http://creativecommons.org/ publicdomain/zero/1.0/) applies to the data made available in this article, unless otherwise stated. 
labor intensive and antibiotic use may favor spread of antimicrobial resistance in pathogens as well as in bacteria belonging to the microbiota [7]. In addition, no vaccine formulation is available which completely protects pigs against $H$. suis infection [8], making development of an alternative therapy desirable [4]. Recently, an inhibitory action of bambermycin, a glycolipid antibiotic, has been described towards $H$. pylori strains [9]. As H. pylori is closely related to $H$. suis, it can be hypothesized that bambermycin might inhibit $H$. suis as well.

Bambermycin (synonyms: flavophospholipol, moenomycin, flavomycin) disrupts bacterial cell wall synthesis [10] and is mainly active against Gram-positive bacteria and to some extent against Gram-negative bacteria such as Pasteurella sp., Brucella sp. and H. pylori [10]. Due to its high molecular weight, bambermycin is not absorbed in the gastro-intestinal tract after oral administration. Until 2006, it was used as a growth promoter in animal feed in the European Union [10]. The growth promoting effects of bambermycin supplementation have been linked with a better equilibrium of the gastro-intestinal microbiota which might be due to a reduced colonization of pathogens, such as Salmonella enterica, Clostridium perfringens and Fusobacterium spp. [10-13], while bacteria considered to have beneficial effects, such as Lactobacillus spp., are not affected. The effect on S. enterica and C. perfringens in the intestinal tract, however, contrasts with the relative insensitivity of these species to bambermycin in vitro [10]. Although bambermycin was frequently used as a feed additive for over 50 years, acquired resistance, transfer of resistance or cross-resistance with other antimicrobials has not yet been reported [10]. It has been described that bambermycin selectively inhibits the growth of Escherichia coli and S. enterica harboring resistance plasmids and decreases the conjugation transfer frequency of resistance plasmids in E. coli, S. enterica and Staphylococcus aureus [14-16].

The main objective of this study was to determine the effect of bambermycin supplementation on the course of a $H$. suis infection, the host response and the gastric microbiota, as all these parameters may be involved in gastric pathology. BALB/c mice were used as an experimental model, as in previous studies we showed that these rodents can easily be colonized with $\mathrm{H}$. suis, resulting in gastritis, epithelial cell hyperproliferation and necrosis of parietal cells [17], which are also representing the main characteristics of a human and porcine infection with this agent.

A second objective of our study was to determine if infeed medication with bambermycin might be useful to control $H$. suis infections in pigs.

\section{Materials and methods}

Minimum inhibitory concentration (MIC) of bambermycin on $\mathrm{H}$. suis strains

H. suis strains

Helicobacter suis strains HS1, HS8 and P13/26 were isolated from the gastric mucosa of pigs from different herds according to the method described by Baele et al. [18]. The strains were shown to be genetically different by multilocus sequence typing [19].

All strains were cultured under biphasic and microaerobic conditions at $37{ }^{\circ} \mathrm{C}$. The biphasic medium consisted of Brucella agar (BD, Franklin Lakes, New Jersey, USA) supplemented with $20 \%$ fetal calf serum (GE Healthcare Life Sciences, Logan, USA), $5 \mathrm{mg}$ amphotericin B/l (Sigma-Aldrich, Saint Louis, Missouri, USA), Campylobacter selective supplement (Oxoid) and Vitox supplement (Oxoid). The $\mathrm{pH}$ of the agar was adjusted to 5 by adding $\mathrm{HCl}$ to a final concentration of approximately $0.05 \%$. Finally, Brucella broth (BD, pH 5) was added on top. Isolates were passaged twice to ensure reliable growth. After incubation, the bacteria were harvested and the concentration was determined using an improved Neubauer counting chamber (Sigma-Aldrich).

\section{Determination of MIC-values of bambermycin}

The MIC of bambermycin for $H$. suis strains HS1, HS8 and P13/26 was determined according to the method described by Vermoote et al. [20]. In brief, a combined Brucella agar and broth dilution method in 24-well plates was used. Twofold dilutions of bambermycin (flavomycin $^{\circledR}$, Huvepharma, Antwerp, Belgium) were added to the agar and broth, with final concentrations ranging from 0.03 to $128 \mu \mathrm{g} / \mathrm{mL}$. In total, $5 \times 10^{7}$ bacte$\mathrm{ria} / \mathrm{mL}$ were added to the broth and incubated during $48 \mathrm{~h}$ under microaerobic conditions. After incubation, $H$. suis was quantified using a quantitative real-time PCR (qPCR) where the ureA gene was amplified. The MIC was determined as the lowest concentration of bambermycin for which $\Delta \mathrm{C}_{\mathrm{t}}$ was at least $1 \mathrm{C}_{\mathrm{t}}$ higher than $\Delta \mathrm{cC}_{\mathrm{t}}$ $\left(\Delta C_{t}=C_{t}\right.$ after incubation $-C_{t}$ before incubation of the bambermycin exposed strains; $\Delta \mathrm{cC}_{\mathrm{t}}=\mathrm{C}_{\mathrm{t}}$ after incubation $-C_{t}$ before incubation of the controls; $C_{t}=$ threshold cycle value) [20]. This is the lowest concentration of bambermycin with at least $50 \%$ less bacterial growth compared to controls without bambermycin.

Staphylococcus aureus ATCC 29213 was included as a reference strain. For this strain, 4 different MIC assays were performed: the broth microdilution procedure according to the CLSI standards, the method described by Butaye et al. [21, 22], the $H$. suis susceptibility assay conditions at $\mathrm{pH} 5$ and the same conditions but at $\mathrm{pH} 7$. 
Effect of bambermycin supplemented diet on a $H$. suis infection in mice

Ethic statement

The in vivo experimental protocol was approved by the Ethical Committee of the Faculty of Veterinary Medicine, Ghent University, Belgium (EC 2015/131; November $\left.17^{\text {th }}, 2015\right)$.

\section{Animals and experimental design}

Forty-eight specific-pathogen-free (SPF) female, 5-weekold BALB/cOlaHsd mice were purchased from Harlan NL (Horst, the Netherlands). All animals tested negative for presence of Helicobacter spp. by the use of a Helicobacter genus specific PCR. The animals were randomly divided into 6 groups, each consisting of 8 mice. The groups were assigned as followed: non- $H$. suis infected mice fed a control diet without bambermycin (H. suisnegative control group; group 1 ), non- $H$. suis infected mice fed a $32 \mathrm{ppm}$ bambermycin supplemented diet (group 2), non- $H$. suis infected mice fed a 64 ppm bambermycin supplemented diet (group 3), H. suis infected mice fed a control diet without bambermycin (H. suispositive control group, group 4), $H$. suis infected mice fed a $32 \mathrm{ppm}$ bambermycin supplemented diet (group 5) and $H$. suis infected mice fed a $64 \mathrm{ppm}$ bambermycin supplemented diet (group 6). For each group, the mice were housed on autoclaved soft wood shavings in 2 separate filter-top cages to minimize cage-effect (i.e. four mice per cage). Drinking water was provided ad libitum. The animals were monitored several times a day during the whole experiment. Enrichment was provided in the form of paper tissues, mouse houses and other homemade available enrichment products. All animals were exposed to a 12:12 light:dark cycle in the same stable under controlled environmental conditions.

One week after arrival, groups 4-6 were inoculated twice with $H$. suis strain HS1 with a 48 h interval. Under brief isoflurane anesthesia and using a ball-tipped gavage needle, $350 \mu \mathrm{L}$ Brucella broth $\left(\mathrm{pH}\right.$ 5) containing $7 \times 10^{7}$ bacteria of HS1 was administered intragastrically. Groups 1-3 received an equal volume of Brucella broth ( $\mathrm{pH} 5$ ). The mice were held in an upright position until they regained consciousness, to minimize the risk of reflux. Starting from 1 week after inoculation, the mice were fed ad libitum a control diet (groups 1 and 4), a diet supplemented with $32 \mathrm{ppm}$ bambermycin (groups 2 and 5) or a diet supplemented with $64 \mathrm{ppm}$ bambermycin (groups 3 and 6). The bambermycin supplemented diets were identical to the control diet, with the exception of being supplemented with 32 or $64 \mathrm{ppm}$ bambermycin and with a variable amount of corn starch, used to correct minor differences in the energy content (Research Diets Inc., New Brunswick, USA). Finally, 9 weeks after the second inoculation, the mice were euthanized by cervical dislocation under deep isoflurane anesthesia. The stomachs were removed and opened along the major curvature. Four longitudinal strips were taken from the forestomach to the duodenum. One strip was fixed in 10\% phosphate-buffered formalin and used for histopathology and immunohistochemistry. The other strips were used for DNA- and RNA-extraction.

\section{Histopathology and immunohistochemistry}

The formalin fixed longitudinal strip from the stomach was embedded in paraffin, sectioned at $5 \mu \mathrm{m}$, rehydrated and deparaffinized.

For each stomach, one of the sections was stained with haematoxylin and eosin, dehydrated and finally mounted with a coverslip for light microscopic evaluation. The severity of gastritis was scored according to the updated Sydney system with some modifications [1, 23]. Both diffuse infiltration with inflammatory cells and the presence of lymphoid aggregates and lymphoid follicles in the mucosa and submucosa were taken into consideration. The diffuse infiltration of mononuclear and polymorphonuclear cells was scored as follows: score 0 for absence of infiltration, score 1 for mild infiltration, score 2 for moderate infiltration and score 3 for marked infiltration. In addition, the formation of lymphoid follicles was scored as follows: score 0 for absence of lymphoid aggregates, score 1 for presence of a small number of lymphoid aggregates $(n<5)$, score 2 for a large number of lymphoid aggregates $(n>5)$ and/or the presence of 1 organized lymphoid follicle and score 3 for the presence of at least 2 organized lymphoid follicles. Based on the scoring of the diffuse infiltration with inflammatory cells and the presence of lymphoid aggregates and lymphoid follicles, an overall gastritis score was obtained. Therefore, the average score was calculated for each group. When an overall score of $0<n \leq 1 ; 1<n \leq 2$ or $2<n \leq 3$ was obtained, the gastritis was considered as mild, moderate and severe, respectively.

The other sections were used to determine $H$. suis colonization density, to study the presence of lymphoepithelial lesions, to analyze the number of infiltrating T-cells, B-cells and macrophages and, finally, to determine the number of parietal cells, necrotic cells and replicating cells. After rehydration and deparaffinization, heat-induced antigen retrieval was performed in citrate buffer ( $\mathrm{pH}$ 6) using a microwave oven. Slides were incubated with $3 \% \mathrm{H}_{2} \mathrm{O}_{2}$ in methanol (5 min) to block endogenous peroxidase activity and $30 \%$ goat serum $(30 \mathrm{~min}$ ) to block non-specific reactions. Negative controls to confirm the specificity of the secondary antibodies were obtained by incubating the sections without the primary antibodies. 
Helicobacter suis colonization was visualized using a polyclonal genus-specific rabbit anti- $H$. pylori antibody (1/320; DakoCytomation, Glostrup, Denmark). For detection of infiltrating $\mathrm{T}$ - and B-cells, a polyclonal rabbit anti-CD3 antibody (1/100; DakoCytomation, Glostrup, Denmark) and a polyclonal rabbit anti-CD20 antibody (1/25; Thermo Scientific, Fremont, USA) were used, respectively. Incubation with primary antibodies directed against Helicobacter, CD3 and CD20 was followed by incubation with a biotinylated goat anti-rabbit IgG antibody (1/500; DakoCytomation). After rinsing, the sections were incubated with a streptavidin-biotin-HRP complex (Agilent Technologies, Santa Clara, California, USA) and the color was developed with diaminobenzidine tetrahydrochloride (DAB) and $\mathrm{H}_{2} \mathrm{O}_{2}$.

To determine the infiltrating macrophages, a primary antibody directed against the F4/80 surface marker (1/50; Santa Cruz Biotechnology, Santa Cruz, USA) was used. Detection was done using a rat $\mathrm{ABC}$ staining system (Santa Cruz Biotechnology). Apoptotic cells were identified by immunohistochemical staining using a rabbit polyclonal antibody directed against active caspase-3 and an anti-rabbit HRP-AEC cell and tissue staining kit (R\&D Systems, Minneapolis, USA). Replicating cells were identified using a mouse monoclonal anti-Ki67 antibody (1/25; Novocastra Laboratories Ltd, Newcastle upon Tyne, UK) and a biotinylated goat anti-mouse IgG antibody (1/200; DakoCytomation). Subsequent visualization was done as described for CD3 and CD20 staining. Parietal cells were identified by immunohistochemical staining for the hydrogen potassium ATPase using a mouse monoclonal antibody (1/200; Abcam Ltd, Cambridge, UK) and a biotinylated goat anti-mouse IgG antibody (1/200; DakoCytomation). Subsequent visualization was done as described for CD3 and CD20 staining. A monoclonal mouse anti-cytokeratin antibody (1/50; DakoCytomation) was used to highlight lymphoepithelial lesions.

Helicobacter suis colonization density was scored according to the updated Sydney system [23]. T-cells, B-cells, macrophages, apoptotic cells, replicating cells and parietal cells were counted in five randomly chosen high power fields (magnification: $400 \times$ ). The average number of positive cells per high power field was then calculated for each mouse.

\section{Quantification of $H$. suis by real time $P C R$}

DNA was extracted from the second gastric tissue strip using the Isolate II Genomic DNA Kit ${ }^{\circledR}$ (Bioline, Taunton, USA), according to the instructions of the manufacturer. The presence of $H$. suis DNA was determined using a species-specific, quantitative real time (RT)-PCR based on the ureA gene [24]. The copy number of the obtained amplicons was calculated and converted to the number of $H$. suis bacteria per $\mathrm{mg}$ gastric tissue, by including Tenfold dilutions of an external standard consisting of a 1236 bp segment of the ure $A B$ gene cluster from $H$. suis strain HS5, as described previously [25].

\section{Expression of markers for inflammation and gastric acid secretion}

RNA was extracted from the third gastric tissue strip using the RNeasy Mini $\mathrm{Kit}^{\circledR}$ (Qiagen, Hilden, Germany), according to the manufacturer's instructions. The obtained RNA concentrations were measured using a NanoDrop ${ }^{\circledR}$ spectrophotometer (Isogen Life Science, Utrecht, The Netherlands), after which the concentration of each sample was adjusted to $1 \mu \mathrm{g} / \mu \mathrm{L}$, followed by cDNA synthesis using the iScript ${ }^{\mathrm{TM}}$ cDNA Synthesis Kit (Bio-Rad, California, USA). Expression analysis was then performed for genes encoding host factors involved in gastric acid secretion $(\mathrm{H}+/ \mathrm{K}+\mathrm{ATPase}$, Sonic Hedgehog, KCNQ1, gastrin, the cholinergic muscarinic M3 receptor, somatostatin, the histamine $\mathrm{H} 2$ receptor and the gastrin CCK-B receptor) and in inflammation (IL4, IL-6, IL-8, IL-10, IL-17, Il-1 $\beta$, IFN- $\gamma$ and TNF $\alpha$ ). The housekeeping genes PPIa, H2afz and HPRT were shown to have a stable mRNA expression and therefore included as reference genes [26]. All primer sequences are shown in Additional file 1. The mRNA expression levels of the reference and target genes were quantified using a RTPCR, as described earlier [26]. No-template-control reaction mixtures were included and all samples were run in duplicate. The $\mathrm{C}_{\mathrm{t}}$-values were first normalized to the geometric mean of the $\mathrm{C}_{\mathrm{t}}$-values of the reference genes. Fold changes were calculated using $\Delta \Delta C_{T}$ method with mean of $\mathrm{C}_{\mathrm{t}}$-values from the control groups (groups 1 and 4 ). Finally, for each target gene, the results were expressed as fold changes of the mRNA expression of groups $2-4$ and 5-6 relative to mRNA expression levels of the H. suisnegative control group and the $H$. suis-positive control group, respectively.

\section{Gastric microbiota composition}

DNA was extracted from the fourth gastric tissue strip using the DNeasy Blood \& Tissue Kit ${ }^{\circledR}$ (Qiagen) according to the instructions of the manufacturer. 16S rRNA amplicon pyrosequencing was performed using the Roche GS-Junior Genome Sequencer as described previously by Rodriguez et al. [27]. The obtained $16 \mathrm{~S}$ rRNA sequence reads were processed using MOTHUR (software package v1.35), Pyronoise algorithm and UCHIME algorithm for alignment and clustering, denoising and chimera detection, respectively $[28,29]$. The obtained read sets were compared to a reference dataset of 
aligned sequences of the corresponding region derived from the SILVA database (v1.15) of full-length rRNA sequences implemented in MOTHUR [30]. The final reads were clustered into operational taxonomic units (OTUs) using the nearest neighbor algorithm using MOTHUR with a 0.03 distance unit cutoff. A taxonomic identity was attributed to each OTU by comparison with the SILVA database using a $80 \%$ homogeneity cutoff. When a taxonomic identification lower than $80 \%$ was obtained, the taxonomic level was labelled with the first defined level from higher level followed by the label "unclassified". Finally, the unique sequences for each OTU were compared with the SILVA data set using the BLASTN algorithm. For each OTU, a consensus taxonomic identification was given when less than $1 \%$ of mismatch with the aligned sequence was obtained. In the final metadata table, the following labelling was used: the population is identical to a taxonomically defined species and is labelled "genus_species"; the population is identical to a reference sequence belonging to a still undefined species and is labelled "genus_NCBI accession number"; the sequence is not identical to any known sequence and is labelled with the corresponding OTU number.

In order to determine the effect of $H$. suis and/or bambermycin supplementation on the gastric microbiota composition, different groups were compared with each other:

(i) Helicobacter suis-negative mice without bambermycin supplementation (i.e. group 1) $(n=5)$ vs H. suisnegative mice with bambermycin supplementation (i.e. groups 2-3) ( $n=7)$, H. suis-positive mice without bambermycin supplementation (i.e. group 4) $(n=4)$ and $H$. suis-positive mice with bambermycin supplementation $(n=9)$ (i.e. groups $5-6)$.

(ii) Helicobacter suis-negative mice (i.e. groups 1-3) vs H. suis-positive mice (i.e. groups 4-6).

(iii) Mice without bambermycin supplementation (i.e. groups 1 and 4) vs mice with bambermycin supplementation (i.e. groups $2-3$ and 5-6).

Subsampled datasets were obtained and evaluated in MOTHUR to estimate the richness, microbial diversity and population evenness by using the Chaol estimator, Simpson's reciprocal index and Simpson's evenness index, respectively [31,32]. Population structure and community membership were assessed with MOTHUR using distance matrix based on Bray-Curtis dissimilarity index. Differences in functional profiles of gastric bacterial communities were analyzed by mapping taxa into several phenotypes (i.e. metabolism, Gram staining, sporulation,...) using METAGENassist ${ }^{\circledR}$ [33]. Only the phenotypes detected in more than $50 \%$ of the samples were included for further analysis.

\section{Statistical analysis}

Statistical analysis was performed using SPSS statistics $24^{\circledR}$ (IBM, New York, USA). Differences in histopathology and fold changes of the markers for gastric acid secretion and inflammation were investigated using the non-parametric Kruskal-Wallis test with Bonferroni correction for multiple comparisons. Correlations between histopathology, fold changes and the number of $H$. suis bacteria were examined using the Pearson correlation coefficient. Differences were considered statistically significant at a corrected $P$ value of less than 0.05 .

Statistical differences in microbial diversity, richness and population evenness between the groups were investigated using non-parametric Kruskal-Wallis tests with Tukey post hoc tests using PRISM 7 (Graphpad Software). Using MOTHUR, community composition differences were investigated using Analysis of molecular variance (AMOVA) and homogeneity of molecular variance (HOMOVA). In order to highlight statistical differences in relative bacterial abundance between the groups, non-parametric Kruskal-Wallis tests with Tukey post hoc tests and Benjamini-Hochberg false discovery rate were performed using the STAMP software. Differences were considered statistically significant at a corrected $P$ value of less than 0.05 .

\section{Results}

\section{MIC of bambermycin on $H$. suis strains}

For all investigated $H$. suis strains, the MIC of bambermycin was $8 \mu \mathrm{g} / \mathrm{mL}$. For the reference strain $S$. aureus ATCC 29213 the MIC-value was $0.12 \mu \mathrm{g} / \mathrm{mL}$ when tested according to the method described by Butaye et al. [21, 22] and $0.5 \mu \mathrm{g} / \mathrm{mL}$ when tested according to the CLSI standards, while MIC-values of 1 and $2 \mu \mathrm{g} / \mathrm{mL}$ were obtained using the $H$. suis susceptibility assay conditions at $\mathrm{pH} 7$ and 5, respectively.

\section{Effect of bambermycin supplementation on $H$. suis colonization in mice}

Non- $H$. suis infected groups 1,2 and 3 tested negative for presence of $H$. suis as determined by qPCR and immunohistochemical staining, while all $H$. suis infected groups 4, 5 and 6 tested positive for $H$. suis. In both bambermycin-supplemented diet groups, the number of colonizing $H$. suis bacteria per $\mathrm{mg}$ gastric tissue as well as the colonization density was not altered compared to the $H$. suis-positive control group without bambermycin 
supplementation (Additional file 2). In all $H$. suis infected groups, $H$. suis bacteria were often found in the lumen of the gastric glands and in close proximity of parietal cells (Additional file 3).
Effect of bambermycin supplementation on $\mathrm{H}$. suis-infection associated pathologies in mice Inflammation

An overview of the defined immune cell populations of each group is shown in Figure 1.
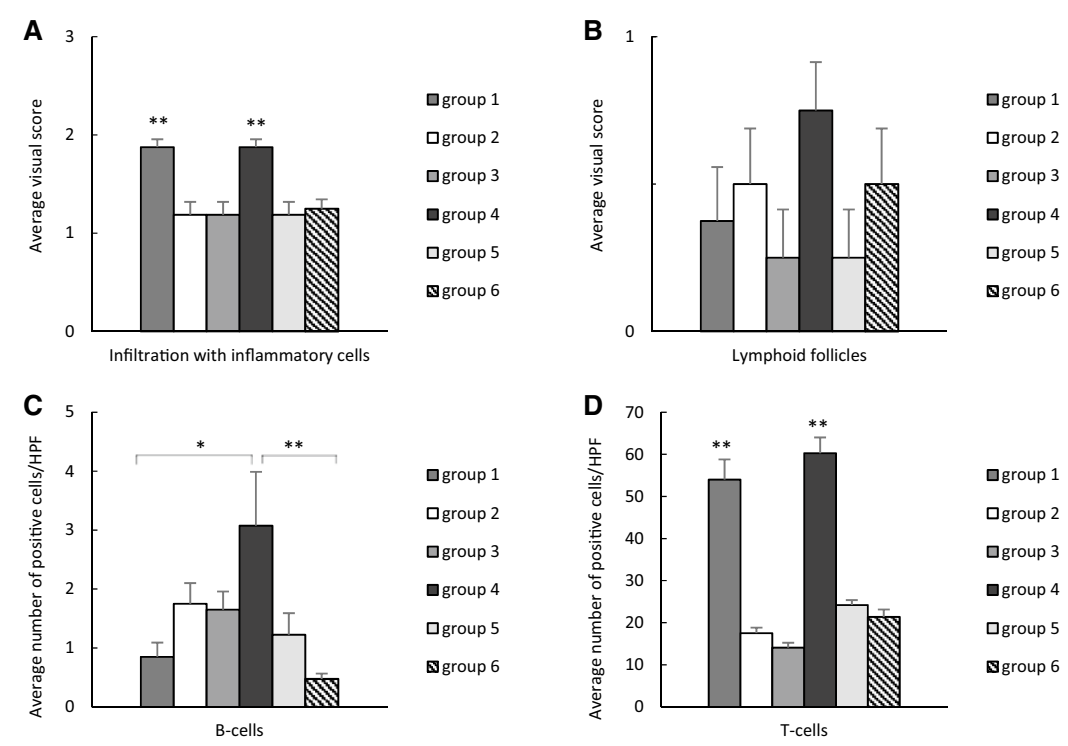

口group 1

口group 2

口group 3

घgroup 4

口group 5

Wroup 6

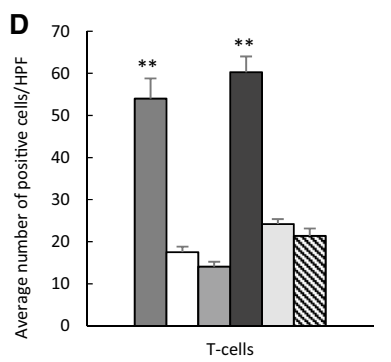

口group 1

口group 2

口group 3

घgroup 4

口group 5

Sgroup 6
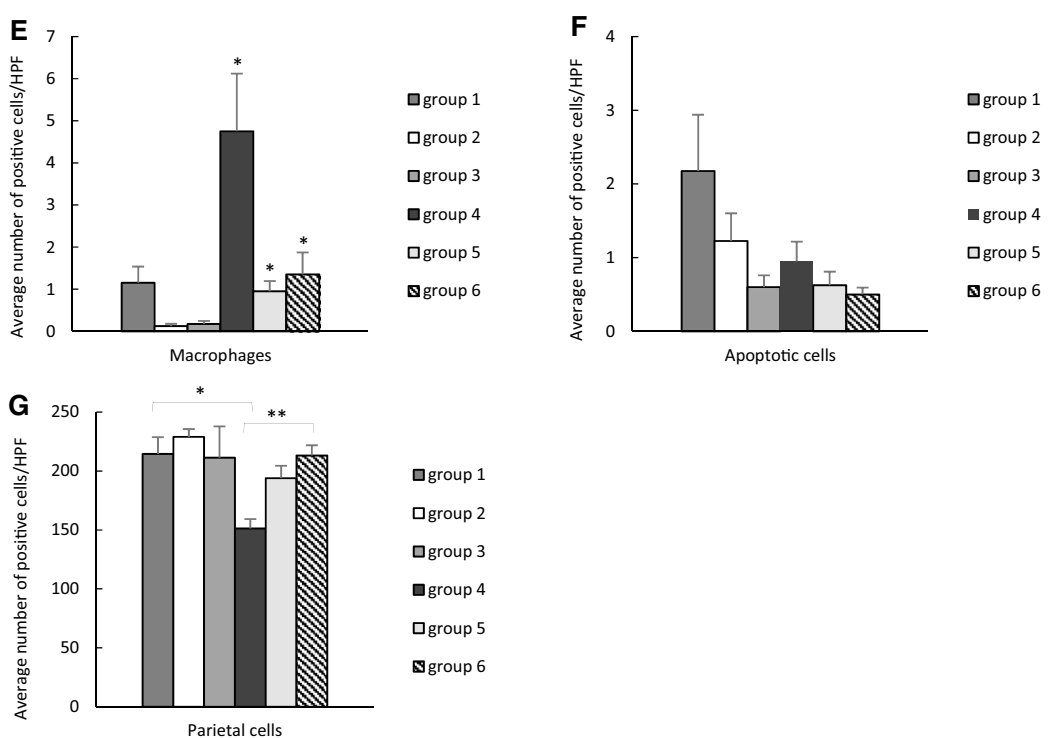

Figure 1 Scores for immune cells, apoptotic cells and parietal cells in bambermycin supplemented and non-supplemented groups. Scores for infiltration with inflammatory cells $(\mathbf{A})$ and lymphoid follicle formation $(\mathbf{B})$; number of infiltrating B-cells $(\mathbf{C})$, T-cells (D), macrophages (E); number of apoptotic cells $(\mathbf{F})$ and parietal cells $(\mathbf{G})$ in bambermycin supplemented and non-supplemented groups. A, B Data are shown as the average scores for infiltration with inflammatory cells/lymphoid follicle formation, with standard deviation, of group 1-6. C-G Data are shown as the average number of positive cells per high power field (HPF), with standard deviation, belonging to a defined cell population, including B-cells, T-cells, macrophages, necrotic cells and parietal cells, of group 1-6. Statistical differences were calculated using the non-parametric Kruskal-Wallis $\mathrm{H}$ test SPSS statistics $24^{\circledR}$. ${ }^{*}$ Significant differences between the groups $(P<0.05),{ }^{* *}$ significant differences between the groups $(P<0.005)$. Group $1=H$. suis-negative control group without bambermycin supplementation; group $2=32 \mathrm{ppm}$ bambermycin supplemented, non- $H$. suis infected group; group $3=64$ ppm bambermycin supplemented, non-H. suis infected group; group $4=H$. suis-positive control group without bambermycin supplementation; group $5=32$ ppm bambermycin supplemented, H. suis infected group; group $6=64$ ppm bambermycin supplemented, H. suis infected group. 
The H. suis-positive control group 4 showed significantly higher numbers of infiltrating B-cells and macrophages compared to the $H$. suis-negative control group $1(P=0.016$ and 0.003 , resp.) (Figure 2). Furthermore, a trend towards a higher number of infiltrating T-cells was seen in all $H$. suis infected groups 4-6 compared to the non-infected groups 1-3, although this was not significant (Figures 1 and 2).

Compared to the H. suis-positive control group 4, a lower number of infiltrating B-cells and macrophages was demonstrated in the $32 \mathrm{ppm}$ bambermycin supplemented, H. suis infected group 5 ( $P=0.068$ and 0.001 , resp.) as well as in the $64 \mathrm{ppm}$ bambermycin supplemented, $H$. suis infected group $6(P=0.003$ and 0.005 , resp.) (Figure 2). In comparison to both groups that did not receive bambermycin (groups 1 and 4), all bambermycin-supplemented diet groups showed significantly lower scores for infiltration with inflammatory cells as well as infiltrating T-cells $(P<0.005$ and $<0.001$, resp.) (Figures 1 and 2$)$. No significant differences were detected in the formation of lymphoid follicles between the groups.

The infiltration with inflammatory cells was positively correlated with the number of infiltrating T-cells and macrophages, while the number of infiltrating T-cells was positively correlated with the number of infiltrating B-cells and macrophages (Additional file 4). No correlations were found between the number of inflammatory cells and the number of colonizing $H$. suis bacteria per mg gastric tissue.

Positive correlations were found between the number of infiltrating macrophages and the expression of IL-8Kc, IL-8Li, IL-10, IL-17, IFN- $\gamma$, TNF- $\alpha$ and IL-1 $\beta$; between the number of infiltrating T-cells and the expression of IL-8Li, IL-10 and IL-1 $\beta$ and between the number of infiltrating B-cells and the expression of IL-4, IL-10 and IL-12 (Additional file 4).

\section{Gastric epithelial cell proliferation and death}

An overview of the defined cell populations of each group is shown in Figure 1.

The H. suis-positive control group 4 showed significantly lower numbers of parietal cells compared to the $H$. suis-negative control group $1(P=0.032)$ (Figures 1 and 2). The $64 \mathrm{ppm}$ bambermycin supplemented, $H$. suisinfected group 6, however, showed significantly higher numbers of parietal cells compared to the $H$. suis-positive control group $4(P=0.038)$. This was also the case for the $32 \mathrm{ppm}$ bambermycin supplemented, $H$. suis infected group 5, although not significant. The number of apoptotic cells and epithelial cell proliferation did not differ between the groups.

Negative correlations were detected between the number of parietal cells and the number of infiltrating T-cells,
B-cells, macrophages and inflammatory cells in general (Additional file 4).

\section{Effect of bambermycin supplementation on the expression of markers for inflammation}

The fold changes of altered markers for inflammation are represented in Figure 3 and Additional file 5.

The H. suis-positive control group 4 showed upregulated expressions of IL-4, IL-6, IL-8M, IL-8Li, IL-10, and TNF- $\alpha$ compared to the $H$. suis-negative control group $1(P=0.091,0.115,0.298,0.002,0.012$ and 0.065 , resp.). In addition, IL-17 and IL-23 were significantly downregulated $(P<0.001)$ (Additional file 5 , Figure 3$)$. Both bambermycin supplemented, $H$. suis infected groups 5 and 6 showed upregulated expressions of IL-4, IL-6 and IL-8Li in comparison with the $H$. suis-negative control group 1 (fold change $=3.24 \pm 0.89,2.95 \pm 0.64$ and $3.25 \pm 0.54 ; P=0.118,0.066$ and 0.002 , resp. for group 5 ; fold change $=1.71 \pm 0.48,2.38 \pm 0.51$ and $3.44 \pm 1.03$; $P=0.970,0.066$ and 0.015 , resp. for group 6), while IL-17 and IL-23 transcript levels were downregulated (fold change $=0.05 \pm 0.02$ and $0.15 \pm 0.05 ; \quad P<0.001$ and $<0.001$, resp. for group 5; fold change $=0.03 \pm 0.01$ and $0.22 \pm 0.07 ; P<0.001$ and $=0.001$, resp. for group 6 ). The expression of TNF- $\alpha$, however, was not altered during bambermycin supplementation in the $H$. suis infected groups 5 and 6 in comparison with the H. suis-negative control group 1. Since positive correlations were found between both, the altered fold changes of IL-4, IL-8Li, IL-17 and IL-23 were more pronounced in mice with a higher number of colonizing $H$. suis bacteria per mg gastric tissue (Additional file 4).

Compared to the $H$. suis-positive control group 4, the mRNA expressions of IL-8M and IFN- $\gamma$ were downregulated in the $32 \mathrm{ppm}$ bambermycin supplemented, H. suis infected group 5 ( $P=0.100$ and 0.015 , resp.), as well as the IL-8M, IL-10 and IFN- $\gamma$ transcript levels in the $64 \mathrm{ppm}$ bambermycin supplemented, $H$. suis infected group $6(P=0.001,0.077,0.253$, resp.).

In both bambermycin supplemented, $H$. suis-negative groups 2 and 3 , the expression of IL- $1 \beta$ was significantly downregulated compared to the $H$. suis-negative control group $1(P=0.028$ and 0.015 , resp.). A similar observation was found for the $64 \mathrm{ppm}$ bambermycin supplemented, $H$. suis-positive group compared to the $H$. suis-negative control group (fold change $=0.72 \pm 0.13$; $P=0.065)$.

\section{Effect of bambermycin supplementation on the expression of markers for gastric acid secretion}

The fold changes of altered markers for gastric acid secretion are presented in Figure 4 and Additional file 6. 

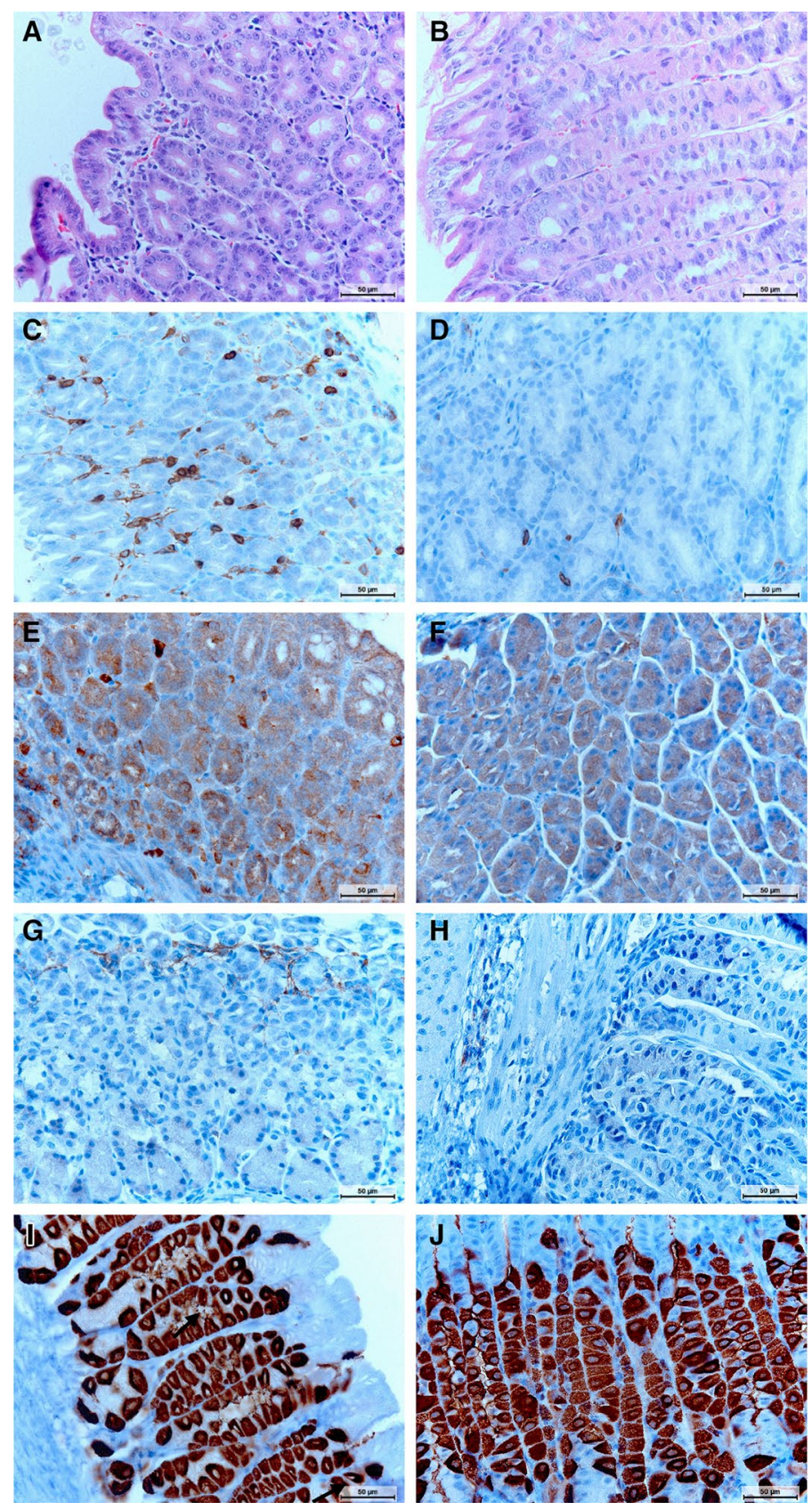

Figure 2 Microscopic visualization of immune cells, apoptotic cells, proliferative cells and parietal cells in the mouse stomach. A, B HE-staining showing infiltration with mononuclear and polymorphonuclear cells in H. suis infected mice without (A) and with bambermycin (B) supplementation. The infiltration is less severe during bambermycin supplementation. Original magnification: $\times 400$. C, D CD3-staining showing infiltration with T-cells (brown) in H. suis infected mice without (C) and with bambermycin $(\mathbf{D})$ supplementation. The infiltration is less severe during bambermycin supplementation. Original magnification: $\times 400$. E, F CD20-staining showing infiltration with B-cells (dark brown) in mice infected with $(\mathbf{E})$ and without $H$. suis $(\mathbf{F})$. The infiltration is more severe during $H$. suis infection. Original magnification: $\times 400$. G, $\mathbf{H}$ F4/80 staining showing infiltration with macrophages (brown) in mice infected with $(\mathbf{G})$ and without $(\mathbf{H})$ H. suis. The infiltration is more severe during H. suis infection. Original magnification: $\times 400$. I, J H+/K+ATPase-staining showing parietal cells (brown) in mice infected with (I) and without (J) H. suis. The number of parietal cells is lower during H. suis infection. H. suis bacteria can be seen in the lumen of the gastric glands (arrow). Original magnification: $\times 400$. 


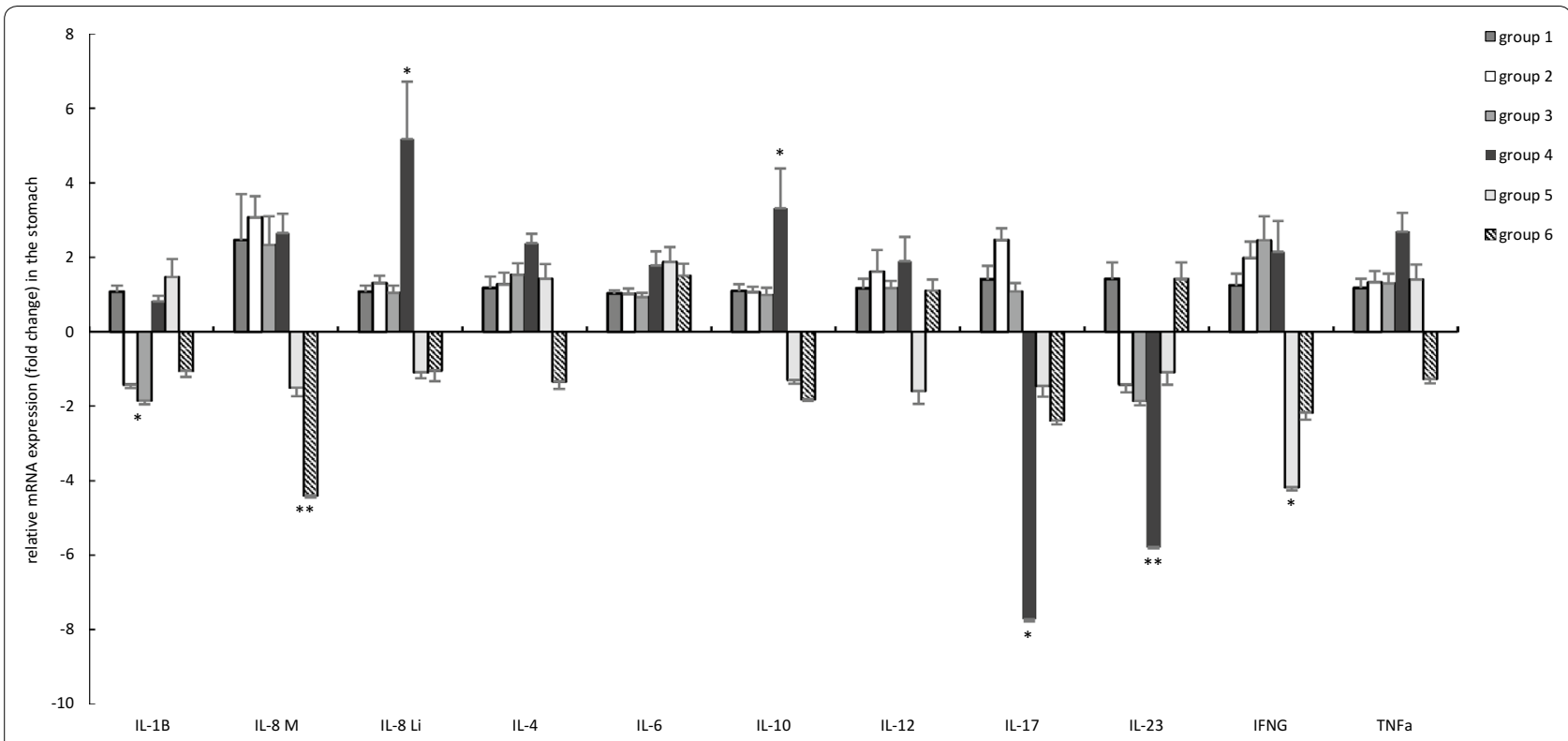

Figure 3 General overview of relative fold changes of altered markers for inflammation in the bambermycin-supplemented and nonsupplemented groups. The data are presented as fold changes in gene expression normalized to 3 reference genes and relative to control groups 1 and 4 (i.e. group 2-4 relative to group 1 and group 5-6 relative to group 4) which are considered as 1. The fold changes are shown as means with the standard error of the mean. Statistical differences were calculated using the non-parametric Kruskal-Wallis H test SPSS statistics $24^{\circledR}$. ${ }^{*}$ Significant differences with the control group $(P<0.05)$, ${ }^{* *}$ significant differences with the control group $(P<0.005)$. Group $1=H$. suis-negative control group without bambermycin supplementation; group $2=32 \mathrm{ppm}$ bambermycin supplemented, non-H. suis infected group; group $3=64$ ppm bambermycin supplemented, non-H. suis infected group; group $4=$ H. suis-positive control group without bambermycin supplementation; group $5=32$ ppm bambermycin supplemented, H. suis infected group; group $6=64$ ppm bambermycin supplemented, H. suis infected group.

In summary, the $H$. suis-positive control group 4 showed upregulated expressions of KCNQ1 and the CCK-B receptor in comparison with the $H$. suis-negative control group $1(P=0.119$ and 0.032 , resp.). In addition, the bambermycin supplemented groups 2-3 and 5 showed upregulated expressions of $\mathrm{H}+/ \mathrm{K}$ ATPase, Sonic Hedgehog, KCNQ1, M3 receptor and/or CCK-B receptor in comparison with the $H$. suis-negative and/or positive control groups 1 and 4 (Figure 4, Additional file 6).

Positive correlations were found between the altered expressions of KCNQ1, CCK-B receptor and somatostatin and the expressions of IL-8 and IL-10 (Additional file 4).

\section{Effect of bambermycin supplementation and/or $\mathrm{H}$. suis} infection on the gastric microbiota composition in mice Despite several attempts, including altering the number of pyrosequencing cycles, no sequencing reads could be obtained from three mice of the $H$. suis-negative control group 1; 5 of group 2; 4 of group 3; 4 of group 4; 4 of group 5 and 3 of group 6 . The other 25 samples yielded sufficient reads after pyrosequencing and were selected for further analysis. Pyrosequencing yielded between 400 and 4000 reads per sample (Additional file 7). A total of 70118 final reads were attributed to 1290 species level OTUs for the 25 samples. Chimeric sequences represented $20 \%$ of the total sequencing reads and were thus excluded from the analysis.

In general, total bacterial community analysis showed that the most dominant phylum in the murine stomach was Firmicutes contributing up to $92 \%$, followed by Bacteroides (4\%) and Proteobacteria (4\%). The relative abundance of other phyla was below $0.1 \%$. On the family level, following populations (i.e.>0.5\%) were dominant in the murine stomach: Lactobacillaceae (63\%), Clostridiaceae (9\%), Erysipelotrichaceae (6\%), Streptococcaceae (4\%) and Porphyromonadaceae (4\%). The major genera (i.e. $>0.5 \%$ ) were Lactobacillus (63\%), Clostridium sensu stricto (9\%), unclassified Erysipelotrichaceae (5\%), Lactococcus (4\%), Parabacteroides (4\%) and Turicibacter (2\%). In two bambermycin supplemented, $H$. suis-positive mice (i.e. one of the $32 \mathrm{ppm}$ supplemented group 5 and one of 


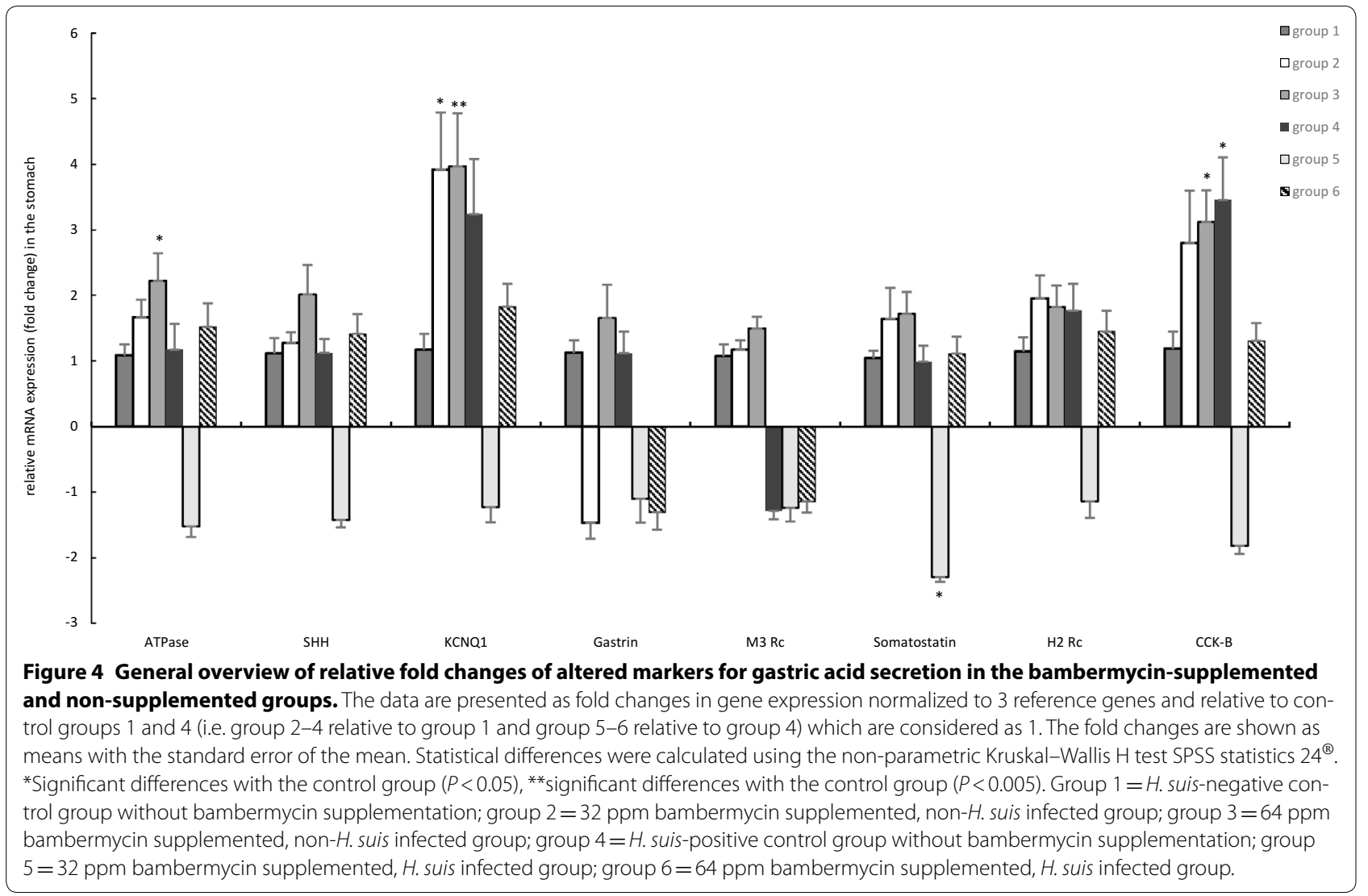

the 64 ppm supplemented group 6), however, Proteobacteria represented the major phylum $(97 \%)$. Helicobacteriaceae (11\%) and Helicobacter (11\%) also represented dominant populations in the murine stomach, but only in the $H$. suis-positive groups $4-6$. The average gastric bacterial community composition at the phylum, family and genus level present in the different groups is represented in Figures $5 \mathrm{~A}-\mathrm{C}$, while the bacterial community composition of each individual mice is shown in Additional files $8 \mathrm{~A}-\mathrm{C}$.

Bambermycin supplementation and/or infection with $H$. suis had no effect on microbial diversity, richness and population evenness (Additional files $9 \mathrm{~A}-\mathrm{C}$ ). Furthermore, Unifrac weighted analysis as well as AMOVA and HOMOVA did not reveal significant differences regarding community structure and composition of the groups. Population structure and community membership, as determined by Bray-Curtis dissimilarity index, was also not different between the groups.

Phenotypic analysis of the murine gastric microbiota in general revealed the presence of 12 metabolic phenotypes of which ammonia oxidizer, dehalogenation, chitin degradation, xylan degrader, sulfide oxidizer and dinitrogen-fixing were the most abundant, each accounting for 78, 78, 14, 7, 7 and $6 \%$ of the gastric bacterial community, respectively. In the gastric microbiota of all mice, non-sporulating and Gram-positive bacteria were more abundant than sporulating and Gram-negative bacteria, respectively ( $70 \%$ vs $10 \%$ and $72 \%$ vs $0.5 \%$, respectively). Presence of $H$. suis infection did not influence the phenotypic features of the gastric bacterial community. The bambermycin supplemented groups, however, showed significant lower abundance of sporulating and chitin degrading bacteria compared to the groups without bambermycin supplementation ( $3.0 \%$ vs $19.7 \%, P=0.027$ and $7.9 \%$ vs $23.7 \%, P=0.027$, respectively).

The taxa Helicobacteriaceae, Helicobacter and H. suis at family, genus and species level, respectively, were only present in the $H$. suis infected groups $(11 \%, P<0.05)$. Infection with $H$. suis did not influence the relative abundance of other taxa at phylum, family or genus level. At species level, Christensenella sp. EF603775 was only present in the $H$. suis-negative mice $(0.041 \%)$, although this difference was not significant $(P=0.223)$. In general, mice that received bambermycin (i.e. groups $2,3,5$ and 6 ) showed a relative lower abundance of the following taxa: 


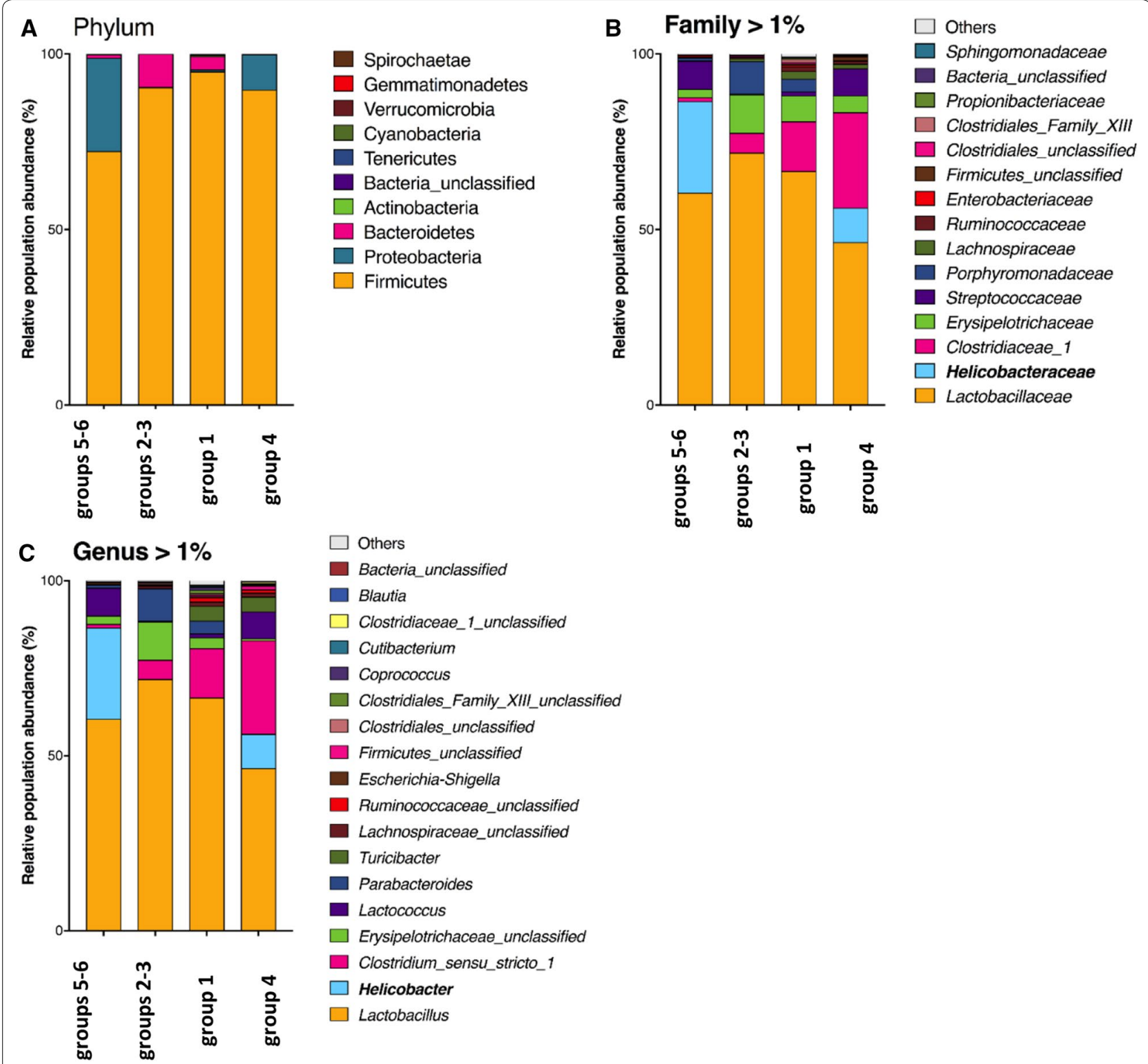

Figure 5 Average gastric bacterial community compositions present in the bambermycin-supplemented and non-supplemented groups. The cumulated histograms show the relative abundance of the identified taxa at phylum (A), family (B) or genus (C) level. At family and genus level, taxa with a relative abundance $<1 \%$ are merged in the category "others". Group $1=$ H. suis-negative control group without bambermycin supplementation; group $2=32 \mathrm{ppm}$ bambermycin supplemented, non-H. suis infected group; group $3=64$ ppm bambermycin supplemented, non-H. suis infected group; group $4=H$. suis-positive control group without bambermycin supplementation; group $5=32$ ppm bambermycin supplemented, H. suis infected group; group $6=64$ ppm bambermycin supplemented, H. suis infected group. The unclassified populations correspond to defined groups of the genus level for which a taxonomical classification assignation to the genus cannot be attributed. These populations are therefore labelled with the first defined superior hierarchical taxonomic level followed by"_unclassified" to prevent confusion.

- At phylum family, genus and species level: Firmicutes_ unclassified.

- At phylum, family and genus level: Bacteria_unclassified.

- At family level: Clostridiaceae_1.
- At genus level: Clostridiaceae_1_unclassified, Coprococcus, Turicibacter, Clostridium_sensu_stricto_1.

- At species level: Coprococcus_EF099198, Coprococcus_ 16S_OTU119, Clostridiales_Family_XIII_AB702776, Clostridiales_Family_XIII_16S_OTU162, Clostridi- 
ales_Family_XIII_EF604613, Clostridiaceae_1_16S_ OTU75, Clostridiaceae_1_16S_OTU107, Firmicutes 16S_OTU195, Firmicutes_16S_OTU37, Firmicutes_ 16S_OTU43, Firmicutes_16S_OTU594, Turicibacter_ EF406660, Turicibacter_DQ015666 and Turicibacter EF406615 (Additional file 10).

The relative abundance of these taxa did not differ between the groups receiving different doses of in-feed bambermycin medication.

Interestingly, the relative abundance of these taxa was positively correlated with infiltration with inflammatory cells, T-cells, B-cells and macrophages as well as the expression of IFN- $\gamma$, gastrin and somatostatin, while negatively correlated with the number of parietal cells and the expression of $\mathrm{H} 2$ receptor, CCK- $\mathrm{B}$ receptor, $\mathrm{H}+$ / $\mathrm{K}+\mathrm{ATPase}$ and KCNQ1 (Additional file 11).

\section{Discussion}

In the present study, an infection with $H$. suis elicited increased infiltration with inflammatory cells such as B-cells, macrophages and T-cells. These findings are in line with previous studies where $H$. suis infection was associated with development of severe gastritis in experimentally and naturally infected pigs and experimentally infected mice and Mongolian gerbils [17, 34, 35]. Since macrophages produce several factors, such as IL-10, which provoke a Th2 response [36] and since B-cells promote the expansion of Th2 cells [37], infiltration with these inflammatory cells may have contributed to the observed Th2 response in $H$. suis infected mice.

The expression of IL-17, however, was downregulated in the H. suis infected mice, in contrast with other studies where a clear IL-17 upregulation and a mixed Th2/Th17 response was present in $H$. suis infected mice $[17,26,34$, $38,39]$. This might be caused by the absence of an IL-23 upregulation, a cytokine stimulating Th17 cell expansion [40]. As Flahou et al. showed an inverse correlation between the $H$. suis colonization rate and the expression of IL-17 [26], the observed high number of colonizing $H$. suis bacteria per mg gastric tissue might have contributed to the downregulation of IL-17. Still, it is not clear why a Th17 response was absent in this study.

BALB/c mice experimentally infected with $H$. pylori and C57BL/6 mice experimentally infected with $\mathrm{H}$. felis, showed a decreased number of Lactobacillus spp. and an increased number of Clostridium spp., Bacteroides spp., Prevotella spp., Eubacterium spp., Ruminococcus spp., Streptococcus spp. and E. coli in their stomach [41, 42]. In another study, however, H. pylori infected C57BL/6 mice did not show gastric microbiota alterations [43]. In the present study, $H$. suis infection was not associated with a shift in the gastric microbiota. Often, the used mouse substrain is not mentioned and although the same strain can be used in different studies, the substrain may be different. These differences in genetic background may contribute to discrepancies between studies [44]. Differences in virulence between Helicobacter sp., stage of infection, and/or influence of the host immune response may also contribute to the discrepancies [45]. In future studies, it would be interesting to investigate a more long term effect of a $H$. suis infection on the gastric microbiota composition.

Since $H$. suis only grows in a biphasic medium with an acidic $\mathrm{pH}$, standard antimicrobial susceptibility assays cannot be used for MIC determinations. The use of the combined agar and broth dilution method [20] may have influenced the results, as Butaye et al. demonstrated that medium composition has a clear impact on bambermycin activity [22]. For example, addition of sheep blood, hemoglobin, albumin, casein and starch as well as variations in $\mathrm{pH}$ and inoculum size affect the activity of bambermycin in vitro [10, 22]. Although for MIC determinations, a strict standardization of the test medium is preferable [22], no optimal medium exists to test bambermycin susceptibility of fastidious micro-organisms, such as $H$. suis. In comparison with the method described by Butaye et al. [22], the MIC endpoints of the reference strain S. aureus ATCC 29213 were 2, 3 and 4 times higher when using the broth microdilution procedure according to CLSI standards and the H. suis susceptibility assay conditions at $\mathrm{pH} 5$ and 7 , respectively. The presence of dextrose, casein and/or other components in Brucella broth may have contributed to the decreased activity of bambermycin.

To the best of our knowledge, no susceptibility data of bambermycin on $H$. suis has been published. In the present study, all $H$. suis strains showed a MIC-value of $8 \mu \mathrm{g} /$ $\mathrm{mL}$. Despite this relatively low MIC endpoint, bambermycin supplementation did not affect the $H$. suis colonization rate in vivo. As no specific clinical breakpoints for bambermycin against $H$. suis are available, prediction of the clinical efficacy based on in vitro testing solely is difficult. This is further complicated by the fact that, even in vitro, different components in the medium such as proteins, starch and lipid substances, may highly influence the antibacterial activity of bambermycin [22]. The gastric environment is far more complex than this 
in vitro environment, making it almost impossible to predict the activity of bambermycin in the stomach from results of MIC determinations.

Despite the absence of an effect on the $H$. suis colonization rate, parietal cell loss during $H$. suis infection was countered when bambermycin was supplemented. A decreased parietal cell mass has also been shown in $H$. suis infected BALB/c mice and Mongolian gerbils [17, 35]. It has been postulated that the loss of parietal cells might be due to a direct interaction of $H$. suis with these cells, since the bacterium can cause degenerative changes and necrosis of parietal cells in pigs, humans and rodent models $[17,46]$ and $H$. suis is able to directly interfere with cultured parietal cells, causing a significant impairment in cell viability [47]. Production of $\gamma$-glutamyl transpeptidase (GGT) by $H$. suis has been linked with these degenerative changes and/or impairment in cell viability [35]. It remains to be determined if bambermycin affects this direct interaction of the bacterium with parietal cells, without influencing its colonization capacity. The inverse correlation seen in the present and other studies [47], between severity of gastric inflammation and the number of parietal cells indicates that not only direct interaction of this bacterium with these host cells, but also inflammation may play a role in parietal cell death in H. suis infected hosts.

Several studies attribute a role to $H$. suis in the development of hyperkeratosis and ulceration of the non-glandular stratified squamous epithelium of the Pars oesophagea of the porcine stomach, although $H$. suis does not colonize this region [4]. It is not completely clear how exactly $H$. suis influences ulcer development, but a recent study indicates that alterations in gastric acid secretion may be involved [3]. Indeed, in $H$. suis infected 6-8 months old pigs with severe hyperkeratosis and erosions of the nonglandular part of the stomach, expression of markers for gastric acid secretion was downregulated. It was hypothesized that decreased gastric acid secretion may affect the composition of the Pars oesophageal microbiota. Indeed, compared to non-infected, 6-8 months old pigs with no obvious lesions, higher numbers of Fusobacterium gastrosuis [48], were detected in the Pars oesophagea of $H$. suis infected pigs with hyperkeratosis and erosions of the Pars oesophagea and downregulated markers for gastric acid secretion. In the present study, bambermycin supplementation resulted in an upregulation of several markers for gastric acid secretion and seemed to counter the parietal cell mass loss during $H$. suis infection. It remains to be determined if bambermycin affects gastric ulcer development in $H$. suis infected pigs.

Although an improved feed conversion and growth has been demonstrated in pigs, cattle and poultry during bambermycin supplementation, the effect on gastrointestinal inflammation had not been investigated before [49-52]. Here, the increased infiltration with macrophages, T-cells and B-cells and upregulated expressions of IL-8M, IL-10, TNF- $\alpha$ and IFN- $\gamma$ in H. suis infected mice was countered when bambermycin was supplemented in the diet, even though this antibiotic apparently did not influence $H$. suis colonization. Similarly, in the stomach of the non- $H$. suis infected mice treated with bambermycin, a decreased T-cell and macrophage infiltration was observed as well as a downregulated expression of the pro-inflammatory cytokine IL-1 $\beta$, further indicating that bambermycin may alter the function of inflammatory cells resulting in a more tempered host immune response. The mechanism behind this anti-inflammatory effect is not clear. It has been hypothesized that macrolides, cyclines and streptogramins may accumulate in phagocytic cells, reducing the production of pro-inflammatory cytokines [53]. Macrolides may also inhibit T-cell maturation and proliferation [54]. It is not known if similar mechanisms are involved for bambermycin.

In the present study, bambermycin supplementation did not cause major shifts in Lactobacillus spp., which have been considered to exert a beneficial effect in the intestinal tract [10]. On the other hand, the presence of bacterial species belonging to Firmicutes, Turicibacter, Coprococcus, Clostridiaceae, Clostridiales family XIII and Clostridium senso stricto 1 taxa was positively correlated with infiltration of inflammatory cells as well as expression of markers for inflammation. This might indicate that some bacteria belonging to these taxa might exert unfavorable effects on the host. Since the relative abundance of these taxa was decreased during bambermycin supplementation, an altered gastric microbiota composition may also have contributed to the antiinflammatory effect of bambermycin. Nevertheless, as most of these species are yet unknown, further research is necessary to confirm or deny this hypothesis. Finally, ammonia oxidizers accounted for $78 \%$ of the gastric bacterial community. These bacteria produce nitric oxide, which may exert damaging effects and contribute to gastritis. It has been shown that bambermycin exhibits an anti-oxidative effect by scavenging free nitric oxide radicals in vitro [55], which may also play a role in the observed anti-inflammatory effect of in-feed bambermycin medication.

In conclusion, bambermycin supplementation did not affect $H$. suis colonization, but did decrease gastric inflammation and inhibited the effects of a $H$. suis infection on parietal cell loss. Not only direct interaction of $H$. suis with parietal cells, but also inflammation may play a role in death of these gastric acid producing cells. 


\section{Additional files}

\section{Additional file 1. List of primers used in quantitative RT-PCR for} gene expression analysis of markers for gastric acid secretion and inflammation.

Additional file 2. Number and colonization density of $\boldsymbol{H}$. suis in groups 4-6. (A) Number of $H$. suis bacteria per mg gastric tissue of group 4-6. Data are shown as $\log 10$ values of the average of number of $H$. suis bacteria per mg tissue with standard deviation. (B) Colonization density of H. suis in the stomach of group 4-6. Data are shown as the average of the colonization score for each group with standard deviation. Group $4=$ H. suis-positive control without bambermycin supplementation; group $5=32 \mathrm{ppm}$ bambermycin supplemented, $H$. suis infected group; group $6=64$ ppm bambermycin supplemented, H. suis infected group.

Additional file 3. Immunohistochemical Helicobacter (A), caspase-3 (B) and KI-67 (C) staining of a mouse stomach, showing H. suis colonization, apoptotic cells and replicating cells, respectively. (A) H. suis bacteria (brown) present in the glands of the antrum of a stomach of a H. suis-positive control mouse not treated with bambermycin. Original magnification: $\times 400$. (B) Apoptotic cells (brown) present in a stomach of a H. suis-negative mouse treated with bambermycin. Original magnification: $\times 400$. (C) Replicating cells (brown) present in a stomach of a H. suisnegative mouse treated with bambermycin. Original magnification: $\times 400$.

Additional file 4. Overview of important correlations. $r=$ Pearson correlation coefficient, calculated using SPSS Statistics $24^{\circledR}$. A r-value close to 1 indicates a strong, positive correlation, whereas a r-value of -1 indicates a strong, negative correlation. $P$-values lower than 0.05 are considered to be significant.

Additional file 5. Overview of the relative fold changes of altered markers for inflammation in the bambermycin-supplemented and non-supplemented groups. The data are presented as fold changes in gene expression normalized to 3 reference genes and relative to control groups 1 and 4 (i.e. group 2-4 relative to group 1 and group 5-6 relative to group 4) which are considered as 1 . The fold changes are shown as means with the standard error of the mean. Statistical differences were calculated using the non-parametric Kruskal-Wallis $\mathrm{H}$ test SPSS statistics $24^{\circledR}$. A P-value lower than 0.05 is considered to be significant. Group $2=32 \mathrm{ppm}$ bambermycin supplemented, non-H. suis infected group; group $3=64$ ppm bambermycin supplemented, non-H. suis infected group; group $4=H$. suis-positive control group without bambermycin supplementation; group $5=32$ ppm bambermycin supplemented, $H$. suis infected group; group $6=64 \mathrm{ppm}$ bambermycin supplemented, H. suis infected group.

Additional file 6. Overview of the relative fold changes of altered markers for gastric acid secretion in the bambermycin-supplemented and non-supplemented groups. The data are presented as fold changes in gene expression normalized to 3 reference genes and relative to control groups 1 and 4 (i.e. group 2-4 relative to group 1 and group 5-6 relative to group 4) which are considered as 1. The fold changes are shown as means with the standard error of the mean. Statistical differences were calculated using the non-parametric Kruskal-Wallis $\mathrm{H}$ test SPSS statistics $24^{\circledR}$. A P-value lower than 0.05 is considered to be significant. Group $2=32 \mathrm{ppm}$ bambermycin supplemented, non-H. suis infected group; group 3 $=64$ ppm bambermycin supplemented, non-H. suis infected group; group $4=H$. suis-positive control group without bambermycin supplementation; group $5=32 \mathrm{ppm}$ bambermycin supplemented, H. suis infected group; group $6=64 \mathrm{ppm}$ bambermycin supplemented, $H$. suis infected group.

Additional file 7. Overview of the number of pyrosequencing reads for each mice. Group $1=$ H. suis-negative control group without bambermycin supplementation; group $2=32$ ppm bambermycin supplemented, non-H. suis infected group; group $3=64$ ppm bambermycin supplemented, non-H. suis infected group; group $4=H$. suis-positive control group without bambermycin supplementation; group $5=32 \mathrm{ppm}$ bambermycin supplemented, H. suis infected group; group $6=64 \mathrm{ppm}$ bambermycin supplemented, H. suis infected group.
Additional file 8. Bacterial community compositions present in the stomach of each individual mice. The cumulated histograms show the relative abundance of the identified taxa at phylum (A), family (B) or genus (C) level. At family and genus level, taxa with a relative abundance $<1 \%$ are merged in the category "others". $M 1_{-}=$group $1=$ H. suis-negative control group without bambermycin supplementation; $\mathrm{M} 2$ $2=32$ ppm bambermycin supplemented, non-H. suis infected group; M3_=group 3 $=64$ ppm bambermycin supplemented, non-H. suis infected group; M4_=group $4=\mathrm{H}$. suis-positive control group without bambermycin supplementation; M5_= group $5=32 \mathrm{ppm}$ bambermycin supplemented, H. suis infected group; M6_ = group $6=64 \mathrm{ppm}$ bambermycin supplemented, H. suis infected group. The unclassified populations correspond to defined groups of the genus level for which a taxonomical classification assignation to the genus cannot be attributed. These populations are therefore labelled with the first defined superior hierarchical taxonomic level followed by "_unclassified" to prevent confusion.

Additional file 9. Overview of the gastric bacterial richness, diversity and evenness of the bambermycin-supplemented and non-supplemented groups. Gastric bacterial richness (A), diversity (B) and evenness (C). The data are represented as box plots: the bottom and top of the box represent the first and the third quartile, the line in the box represents the median and the whiskers represent the minimum and maximum values. Group $1=$ H. suis-negative control group without bambermycin supplementation; group $2=32 \mathrm{ppm}$ bambermycin supplemented, non-H. suis infected group; group $3=64$ ppm bambermycin supplemented, non-H. suis infected group; group $4=H$. suis-positive control group without bambermycin supplementation; group $5=32 \mathrm{ppm}$ bambermycin supplemented, H. suis infected group; group $6=64 \mathrm{ppm}$ bambermycin supplemented, H. suis infected group.

Additional file 10. An overview of the main differences in relative abundance of taxa at phylum, family, genus and species level in the bambermycin-supplemented and non-supplemented groups. The data are presented as the mean relative abundance of the taxa with the standard error of the mean. Statistical differences were calculated using the non-parametric Kruskal-Wallis tests with Tukey post hoc tests and Benjamini-Hochberg False Discovery Rate were performed using STAMP ${ }^{\circledR}$. A $P$-value lower than 0.05 is considered to be significant.

Additional file 11. Overview of important correlations between the gastric bacterial community and the number of inflammatory cells, parietal cells and expression of markers for inflammation and gastric acid secretion. $r$ = Pearson correlation coefficient, calculated using SPSS Statistics $24^{\circledR}$. A r-value close to 1 indicates a strong, positive correlation, whereas a r-value of -1 indicates a strong, negative correlation. $P$-values lower than 0.05 are considered to be significant.

\section{Competing interests}

The authors have the following interests: this work received partial financial support from Huvepharma, Antwerp, Belgium. Co-author Veerle Hautekiet is employed by Huvepharma. There are no products in development or marketed products to declare. This does not alter the authors' adherence to all the Veterinary Research policies on sharing data and materials. The other authors, $\mathrm{CDW}, \mathrm{FH}, \mathrm{BT}, \mathrm{GD}, \mathrm{RD}$ and $\mathrm{BF}$, declare that they have no competing interests.

\section{Authors' contributions}

CDW, FH, BT and BF participated in the design of the study. CDW carried out the experiments, analyzed the data and drafted the manuscript. FH coordinated the study, drafted the manuscript and participated in analysis of the data. BT participated in analysis of the data. All authors read and approved the final manuscript.

\section{Acknowledgements}

This research was partially supported by Huvepharma, under contract number 40014429 and by a grant from the Special Research Fund of Ghent University (BOF), Ghent, Belgium (Grant No. 01D20414). We would like to thank Sofie De Bruyckere for her excellent technical support with the determination of MIC values. We would also like to thank Christian Puttevils for his excellent technical support with the tissue staining. 


\section{Ethics approval and consent to participate}

The in vivo experimental protocol was approved by the Ethical Committee of the Faculty of Veterinary Medicine, Ghent University, Belgium (EC 2015/131; November $17^{\text {th }}, 2015$ )

\section{Author details}

${ }^{1}$ Department of Pathology, Bacteriology and Avian Diseases, Faculty of Veterinary Medicine, Ghent University, Merelbeke, Belgium. ${ }^{2}$ Department of Food Sciences, FARAH, Université de Liège, Avenue de Cureghem 10, 4000 Liège, Belgium. ${ }^{3}$ Huvepharma, Uitbreidingstraat 80, 2600 Antwerp, Belgium.

\section{Publisher's Note}

Springer Nature remains neutral with regard to jurisdictional claims in published maps and institutional affiliations.

\section{Received: 22 January 2018 Accepted: 21 March 2018}

Published online: 10 April 2018

\section{References}

1. De Bruyne E, Flahou B, Chiers K, Meyns T, Kumar S, Vermoote M, Pasmans F, Millet S, Dewulf J, Haesebrouck F, Ducatelle R (2012) An experimental Helicobacter suis infection causes gastritis and reduced daily weight gain in pigs. Vet Microbiol 160:449-454

2. Hellemans A, Chiers K, De Bock M, Decostere A, Haesebrouck F, Ducatelle R, Maes D (2007) Prevalence of "Candidatus Helicobacter suis" in pigs of different ages. Vet Rec 161:189-192

3. De Witte C, Devriendt B, Flahou B, Bosschem I, Ducatelle R, Smet A, Haesebrouck F (2017) Helicobacter suis induces changes in gastric inflammation and acid secretion markers in pigs of different ages. Vet Res 48:34

4. Haesebrouck F, Pasmans F, Flahou B, Chiers K, Baele M, Meyns T, Decostere A, Ducatelle R (2009) Gastric helicobacters in domestic animals and nonhuman primates and their significance for human health. Clin Microbiol Rev 22:202-223

5. De Cooman L, Flahou B, Houf K, Smet A, Ducatelle R, Pasmans F, Haesebrouck $F$ (2013) Survival of Helicobacter suis bacteria in retail pig meat. Int J Food Microbiol 166:164-167

6. Malfertheiner P, Megraud F, O'Morain CA, Atherton J, Axon ATR, Bazzoli F, Gensini GF, Gisbert JP, Graham DY, Rokkas T, El-Omar EM, Kuipers EJ, European Helicobacter Study Group TEHSG (2012) Management of Helicobacter pylori infection-the Maastricht IV/florence consensus report. Gut 61:646-664

7. Vermoote M, Van Steendam K, Flahou B, Smet A, Pasmans F, Glibert P, Ducatelle R, Deforce D, Haesebrouck F (2012) Immunization with the immunodominant Helicobacter suis urease subunit B induces partial protection against $H$. suis infection in a mouse model. Vet Res 43:72

8. Haesebrouck F, Pasmans F, Chiers K, Maes D, Ducatelle R, Decostere A (2004) Efficacy of vaccines against bacterial diseases in swine: what can we expect? Vet Microbiol 100:255-268

9. Tseng YY, Liou JM, Hsu TL, Cheng WC, Wu MS, Wong CH (2014) Development of bacterial transglycosylase inhibitors as new antibiotics: moenomycin A treatment for drug-resistant Helicobacter pylori. Bioorg Med Chem Lett 24:2412-2414

10. Butaye P, Devriese LA, Haesebrouck F (2003) Antimicrobial growth promoters used in animal feed: effects of less well known antibiotics on gram-positive bacteria. Clin Microbiol Rev 16:175-188

11. Stutz MW, Lawton GC (1984) Effects of diet and antimicrobials on growth, feed efficiency, intestinal Clostridium perfringens, and ileal weight of broiler chicks. Poult Sci 63:2036-2042

12. Bolder NM, Wagenaar JA, Putirulan FF, Veldman KT, Sommer M (1999) The effect of flavophospholipol (Flavomycin) and salinomycin sodium (Sacox) on the excretion of Clostridium perfringens, Salmonella enteritidis, and Campylobacter jejuni in broilers after experimental infection. Poult Sci 78:1681-1689

13. Edwards JE, Bequette BJ, McKain N, McEwan NR, Wallace RJ (2005) Influence of flavomycin on microbial numbers, microbial metabolism and gut tissue protein turnover in the digestive tract of sheep. Br J Nutr 94:64-70
14. Riedl S, Ohlsen K, Werner G, Witte W, Hacker J (2000) Impact of flavophospholipol and vancomycin on conjugational transfer of vancomycin resistance plasmids. Antimicrob Agents Chemother 44:3189-3192

15. George BA, Fagerberg DJ, Quarles CL, Fenton JM, McKinley GA (1982) Effect of bambermycins on quantity, prevalence, duration, and antimicrobial resistance of Salmonella typhimurium in experimentally infected broiler chickens. Am J Vet Res 43:299-303

16. Van den Bogaard AE, Hazen M, Hoyer M, Oostenbach P, Stobberingh EE (2002) Effects of flavophospholipol on resistance in fecal Escherichia coli and enterococci of fattening pigs. Antimicrob Agents Chemother 46:110-118

17. Flahou B, Haesebrouck F, Pasmans F, D'Herde K, Driessen A, van Deun K, Smet A, Duchateau L, Chiers K, Ducatelle R (2010) Helicobacter suis causes severe gastric pathology in mouse and Mongolian gerbil models of human gastric disease. PLoS One 5:e14083

18. Baele M, Decostere A, Vandamme P, Ceelen L, Hellemans A, Mast J, Chiers K, Ducatelle R, Haesebrouck F (2008) Isolation and characterization of Helicobacter suis sp. nov. from pig stomachs. Int J Syst Evol Microbiol 58:1350-1358

19. Liang J, Ducatelle R, Pasmans F, Smet A, Haesebrouck F, Flahou B (2013) Multilocus sequence typing of the porcine and human gastric pathogen Helicobacter suis. J Clin Microbiol 51:920-926

20. Vermoote M, Pasmans F, Flahou B, Van Deun K, Ducatelle R, Haesebrouck F (2011) Antimicrobial susceptibility pattern of Helicobacter suis strains. Vet Microbiol 153:339-342

21. Butaye P, Devriese LA, Haesebrouck F (1998) Effects of different test conditions on MICs of food animal growth-promoting antibacterial agents for enterococci. J Clin Microbiol 36:1907-1911

22. Butaye P, Devriese LA, Haesebrouck F (2000) Influence of different medium components on the in vitro activity of the growth-promoting antibiotic flavomycin against enterococci. J Antimicrob Chemother 46:713-716

23. Dixon MF, Genta RM, Yardley JH, Correa P (1996) Classification and grading of gastritis. The updated Sydney system. International workshop on the histopathology of gastritis, Houston 1994. Am J Surg Pathol 20:1161-1181

24. Blaecher C, Smet A, Flahou B, Pasmans F, Ducatelle R, Taylor D, Weller C, Bjarnason I, Charlett A, Lawson AJ, Dobbs RJ, Dobbs SM, Haesebrouck F (2013) Significantly higher frequency of Helicobacter suis in patients with idiopathic parkinsonism than in control patients. Aliment Pharmacol Ther 38:1347-1353

25. O'Rourke JL, Solnick JV, Neilan BA, Seidel K, Hayter R, Hansen LM, Lee A (2004) Description of "Candidatus Helicobacter heilmannii" based on DNA sequence analysis of $16 \mathrm{~S}$ rRNA and urease genes. Int I Syst Evol Microbiol 54:2203-2211

26. Flahou B, Van Deun K, Pasmans F, Smet A, Volf J, Rychlik I, Ducatelle R, Haesebrouck F (2012) The local immune response of mice after Helicobacter suis infection: strain differences and distinction with Helicobacter pylori. Vet Res 43:75

27. Rodriguez C, Taminiau B, Brévers B, Avesani V, Van Broeck J, Leroux A, Gallot M, Bruwier A, Amory H, Delmée M, Daube G (2015) Faecal microbiota characterisation of horses using 16 rdna barcoded pyrosequencing, and carriage rate of Clostridium difficile at hospital admission. BMC Microbiol 15:181

28. Haas BJ, Gevers D, Earl AM, Feldgarden M, Ward DV, Giannoukos G, Ciulla D, Tabbaa D, Highlander SK, Sodergren E, Methé B, DeSantis TZ, Petrosino JF, Knight R, Birren BW, Human Microbiome Consortium JF (2011) Chimeric 16S rRNA sequence formation and detection in Sanger and 454-pyrosequenced PCR amplicons. Genome Res 21:494-504

29. Schloss PD, Westcott SL, Ryabin T, Hall JR, Hartmann M, Hollister EB, Lesniewski RA, Oakley BB, Parks DH, Robinson CJ, Sahl JW, Stres B, Thallinger GG, Van Horn DJ, Weber CF (2009) Introducing mothur: open-source, platform-independent, community-supported software for describing and comparing microbial communities. Appl Environ Microbiol 75:7537-7541

30. Pruesse E, Quast C, Knittel K, Fuchs BM, Ludwig W, Peplies J, Glöckner FO (2007) SILVA: a comprehensive online resource for quality checked and aligned ribosomal RNA sequence data compatible with ARB. Nucleic Acids Res 35:7188-7196 
31. Chao A, Shen TJ (2003) Nonparametric estimation of Shannon's index of diversity when there are unseen species in sample. Environ Ecol Stat 10:429-443

32. Chao A, Bunge J (2002) Estimating the number of species in a stochastic abundance model. Biometrics 58:531-539

33. Arndt D, Xia J, Liu Y, Zhou Y, Guo AC, Cruz JA, Sinelnikov I, Budwill K, Nesbo CL, Wishart DS (2012) METAGENassist: a comprehensive web server for comparative metagenomics. Nucleic Acids Res 40:W88-W95

34. Bosschem I, Bayry J, De Bruyne E, Van Deun K, Smet A, Vercauteren G, Ducatelle R, Haesebrouck F, Flahou B (2015) Effect of different adjuvants on protection and side-effects induced by Helicobacter suis whole-cell lysate vaccination. PLoS One 10:e0131364

35. Zhang G, Ducatelle R, De Bruyne E, Joosten M, Bosschem I, Smet A, Haesebrouck F, Flahou B (2015) Role of $\gamma$-glutamyltranspeptidase in the pathogenesis of Helicobacter suis and Helicobacter pylori infections. Vet Res 46:31

36. Duque GA, Descoteaux A (2014) Macrophage cytokines: involvement in immunity and infectious diseases. Front Immunol 5:491

37. Gajewski TF, Pinnas M, Wong T, Fitch FW (1991) Murine Th1 and Th2 clones proliferate optimally in response to distinct antigen-presenting cell populations. J Immunol 146:1750-1758

38. Bosschem I, Flahou B, Van Deun K, De Koker S, Volf J, Smet A, Ducatelle R, Devriendt B, Haesebrouck F (2017) Species-specific immunity to Helicobacter suis. Helicobacter 22:e12375

39. Bosschem I, Flahou B, Bakker J, Heuvelman E, Langermans JAM, De Bruyne E, Joosten M, Smet A, Ducatelle R, Haesebrouck F (2016) Comparative virulence of in vitro-cultured primate- and pig-associated Helicobacter suis strains in a BALB/c mouse and a Mongolian gerbil model. Helicobacter 22:e12349

40. Ouyang W, Kolls JK, Zheng Y (2008) The biological functions of Thelper 17 cell effector cytokines in inflammation. Immunity 28:454-467

41. Aebischer T, Fischer A, Walduck A, Schlötelburg C, Lindig M, Schreiber S, Meyer TF, Bereswill S, Göbel UB (2006) Vaccination prevents Helicobacter pylori-induced alterations of the gastric flora in mice. FEMS Immunol Med Microbiol 46:221-229

42. Schmitz JM, Durham CG, Schoeb TR, Soltau TD, Wolf KJ, Tanner SM, McCracken VJ, Lorenz RG (2011) Helicobacter felis-associated gastric disease in microbiota-restricted mice. J Histochem Cytochem 59:826-841

43. Tan MP, Kaparakis M, Galic M, Pedersen J, Pearse M, Wijburg OLC, Janssen PH, Strugnell RA (2007) Chronic Helicobacter pylori infection does not significantly alter the microbiota of the murine stomach. Appl Environ Microbiol 73:1010-1013

44. Fontaine DA, Davis DB (2016) Attention to background strain is essential for metabolic research: C57BL/6 and the international knockout mouse consortium. Diabetes 65:25-33

45. Suzuki TA, Nachman MW (2016) Spatial heterogeneity of gut microbial composition along the gastrointestinal tract in natural populations of house mice. PLoS One 11:e0163720

46. Joo M, Ji EK, Sun HC, Kim H, Chi JG, Kim KA, Jeon HY, June SL, Moon YS, Kim KM (2007) Helicobacter heilmannii-associated gastritis: clinicopathologic findings and comparison with Helicobacter pylori-associated gastritis. J Korean Med Sci 22:63-69

47. Zhang G, Ducatelle R, Mihi B, Smet A, Flahou B, Haesebrouck F (2016) Helicobacter suis affects the health and function of porcine gastric parietal cells. Vet Res 47:101

48. De Witte C, Flahou B, Ducatelle R, Smet A, De Bruyne E, Cnockaert M, Taminiau B, Daube G, Vandamme P, Haesebrouck F (2016) Detection, isolation and characterization of Fusobacterium gastrosuis sp. nov. colonizing the stomach of pigs. Syst Appl Microbiol 40:42-50

49. Parks CW, Grimes JL, Ferket PR, Fairchild AS (2001) The effect of mannanoligosaccharides, bambermycins, and virginiamycin on performance of large white male market turkeys. Poult Sci 80:718-723

50. Hagsten I, Grant RJ, Meade RJ, Kelley RO (1980) Effect of bambermycins and tylosin on performance of growing-finishing swine. J Anim Sci 50:484-489
51. Sharifi SD, Dibamehr A, Lotfollahian $H$, Baurhoo B (2012) Effects of flavomycin and probiotic supplementation to diets containing different sources of fat on growth performance, intestinal morphology, apparent metabolizable energy, and fat digestibility in broiler chickens. Poult Sci 91:918-927

52. De Schrijver R, Fremaut D, Claes B (1991) Flavomycin effects on performance of beef bulls and nutrient digestibility in wethers. Dtsch Tierarzt Wochenschr 98:47-50

53. Niewold TA (2007) The nonantibiotic anti-inflammatory effect of antimicrobial growth promoters, the real mode of action? A hypothesis. Poult Sci 86:605-609

54. Menconi A, Bielke LR, Hargis BM, Tellez G (2014) Immuno-modulation and anti-inflammatory effects of antibiotic growth promoters versus probiotics in the intestinal tract. J Microbiol Res Rev 2:62-67

55. Kabploy Krittika, Bunyapraphatsara N, Morales Noppawan Phumala, Paraksa N (2015) Study of free radical scavenging activity of antibiotic growth promoters flavophospholipol and avilamycin. Thai J Vet Med 45:389-398

56. Fukuhara S, Matsuzaki J, Tsugawa H, Masaoka T, Miyoshi S, Mori H, Fukushima Y, Yasui M, Kanai T, Suzuki H (2014) Mucosal expression of aquaporin-4 in the stomach of histamine type 2 receptor knockout mice and Helicobacter pylori-infected mice. J Gastroenterol Hepatol 29:53-59

57. Takaishi S, Wang TC (2007) Gene expression profiling in a mouse model of Helicobacter-induced gastric cancer. Cancer Sci 98:284-293

58. Osawa H, Kita H, Ohnishi H, Mutoh H, Ishino Y, Satoh K, Sugano K (2005) Histamine-2 receptor expression in gastric mucosa before and after Helicobacter pylori cure. Aliment Pharmacol Ther 21(Suppl 2):92-98

59. Kitazawa T, Asakawa K, Nakamura T, Teraoka H, Unno T, Komori S, Yamada M (2009) M3 muscarinic receptors mediate positive inotropic responses in mouse atria: a study with muscarinic receptor knockout mice. J Pharmacol Exp Ther 330:487-493

60. Du GM, Liu MJ, Parvizi N, Zhao RQ (2013) Ectopic expression of ghrelin affects gastric $\mathrm{H}+-\mathrm{K}+$-ATPase activity and expression of GHR/IGF-1 system in weaned mice. Regul Pept 186:12-17

61. Jain RN, Brunkan CS, Chew CS, Samuelson LC (2006) Gene expression profiling of gastrin target genes in parietal cells. Physiol Genom 24:124-132

62. Catrysse L, Farhang Ghahremani M, Vereecke L, Youssef SA, Mc Guire C, Sze M, Weber A, Heikenwalder M, de Bruin A, Beyaert R, van Loo G (2016) A20 prevents chronic liver inflammation and cancer by protecting hepatocytes from death. Cell Death Dis 7:e2250

63. Liu C, Rossi M, Lindén S, Padra M, Blaecher C, Bauwens E, Joosten M, Flahou B, Van den Broeck W, Ducatelle R, Haesebrouck F, Smet A (2016) The Helicobacter heilmannii hofE and hoff genes are essential for colonization of the gastric mucosa and play a role in IL-1beta-induced gastric MUC13 expression. Helicobacter 21:504-522

64. Castiglioni A, Corna G, Rigamonti E, Basso V, Vezzoli M, Monno A, Almada AE, Mondino A, Wagers AJ, Manfredi AA, Rovere-Querini P (2015) FOXP3+T cells recruited to sites of sterile skeletal muscle injury regulate the fate of satellite cells and guide effective tissue regeneration. PLoS One 10:e0128094

65. Huang ZH, Reardon CA, Getz GS, Maeda N, Mazzone T (2015) Selective suppression of adipose tissue apoE expression impacts systemic metabolic phenotype and adipose tissue inflammation. J Lipid Res 56:215-226

66. Ichikawa S, Miyake M, Fujii R, Konishi Y (2012) MyD88 associated ROS generation is crucial for lactobacillus induced IL-12 production in macrophage. PLoS One 7:e35880

67. Lee K, Won HY, Bae MA, Hong JH, Hwang ES (2011) Spontaneous and aging-dependent development of arthritis in NADPH oxidase 2 deficiency through altered differentiation of CD11b+ and Th/Treg cells. Proc Natl Acad Sci U S A 108:9548-9553

68. Tortola L, Rosenwald E, Abel B, Blumberg H, Schäfer M, Coyle AJ, Renauld JC, Werner S, Kisielow J, Kopf M (2012) Psoriasiform dermatitis is driven by IL-36-mediated DC-keratinocyte crosstalk. J Clin Investig 122:3965-3976 